\title{
5-(1-苯基-3-苯基丙基-2-炔基)-2,2-亚戊基-1,3-二噁烷-4,6-二酮 衍生物的有效合成
}

\author{
范乃立 $^{a}$ 许招会 $*, a$ 向正兵 ${ }^{b}$ 肖 强 ${ }^{b}$ 廖传文 ${ }^{*, b}$ \\ ( ${ }^{a}$ 江西师范大学化学化工学院 南昌 330027) \\ ( ${ }^{b}$ 江西省人民医院外科 南昌 330006)
}

\begin{abstract}
摘要 在醋酸铜/铜催化体系作用下, 以醛、2,2-亚戊基-1,3-二噁烷-4,6-二酮和芳基乙炔为原料, 发生包含 Knoevenagel 缩合与共轭加成反应的多组分反应, 有效地合成了 12 种 5-(1-苯基-3-苯基丙基-2-炔基)-2,2-亚戊基-1,3-二噁烷-4,6-二酮 衍生物。该工艺具有收率高 $(65 \%$ \% $88 \%$ )、反应温和、操作简单及过量铜粉还能回收利用等优点.

关键词 多组分反应; 醋酸铜/铜; 2,2-亚戊基-1,3-二噁烷-4,6-二䣳; 5-(1-苯基-3-苯基丙基-2-炔基)-2,2-亚戊基-1,3-二噁 烷-4,6-二酮衍生物
\end{abstract}

\section{Efficent Synthesis of 5-(1-Phenyl-3-phenylprop-2-ynyl)- 2,2-pentylidene-1,3-dioxane-4,6-dione Derivatives}

\author{
Fan, Naili $^{a} \quad \mathrm{Xu}$, Zhaohui*,a $^{*}$ Xiang, Zhengbing $^{b} \quad$ Xiao, Qiang $^{b} \quad$ Liao, Chuanwen $^{*, b}$ \\ ( ${ }^{a}$ Department of Chemistry and Chemical Engineering, Jiangxi Normal University, Nanchang 330027) \\ $\left({ }^{b}\right.$ Department of General Surgery, Jiangxi Province People's Hospital, Nanchang 330006)
}

\begin{abstract}
A convenient and efficient approach for the synthesis of 5-(1-phenyl-3-phenylprop-2-ynyl)-2,2-pentylidene-1,3dioxane-4,6-dione derivatives through a three-component reaction of aldehydes with 2,2-pentylidene-1,3-dioxane-4,6-dione and arylacetylene in the presence of $\mathrm{Cu}(\mathrm{OAc})_{2} \cdot \mathrm{H}_{2} \mathrm{O} / \mathrm{Cu}$ is described with 12 examples. The reaction tolerates a wide range of aldehydes furnishing the products with good to excellent isolated yields $(65 \% \sim 88 \%)$. Additionally, the synthetic protocol has the advantages of wild conditions, simple operation, and excessive copper reused.

Keywords multicomponent reaction; copper acetate/copper; 2,2-pentylidene-1,3-dioxane-4,6-dione; 5-(1-phenyl-3-phenylprop-2-ynyl)-2,2-pentylidene-1,3-dioxane-4,6-dione derivatives
\end{abstract}

\section{Introduction}

$\beta$-Alkynyl Meldrum's acid analogues as an interesting building blocks, have exhibited a variety of biological effects, such as PDE IV inhibitors, TNF inhibitors, GPR40 receptor agonists, and GRP receptor antagonists. ${ }^{[1]}$ The compounds are readily transformed to access diverse $\beta$-alkynyl carbonyl compounds ${ }^{[2]}$ and $\gamma$-butyrolactones ${ }^{[3]}$ distributed widely in a vast of natural bioactive molecules such as cyanobacterin, ${ }^{[4]}$ atranone $\mathrm{F},{ }^{[5]}$ growth regulating regent, ${ }^{[6]}$ and goniobutenolides (Figure 1) ${ }^{[7]}$

The conjugate addition of 5-arylmethylene-1,3-dioxane4,6-dione to terminal alkynes is a clean and effective strategy for new $\mathrm{C}-\mathrm{C}$ bond formation and the assembly of $\beta$-chiral carbonyl compounds. 5-(1-Phenyl-3-phenylprop2-ynyl)-2,2-dimethyl-1,3-dioxane-4,6-diones are commonly synthesized employing two methods of metalated teminal alkynes and in situ generated metal alkynlides (Scheme 1). The first known method for the conjugate addition of alkynes includes the use of boron ${ }^{[8,9]}$ or aluminum alkynylides ${ }^{[10]}$ in the presence of $t-\mathrm{BuMe}_{2} \mathrm{SiOTf}^{[11]}$ as an activator under the conditions of rigorous exclusion of oxygen and moisture (Scheme 1a). From a practical point of view, the conjugate addition of in situ generated metal alkynylides is of great attraction, as it is accompolished in a single synthetic operation. In a series of elegant papers, Carreira ${ }^{[12]}$ and Aponick et al. ${ }^{[13]}$ reported the direct conjugate addition of in situ generated Cu-acetylides to Mel-

\footnotetext{
* Corresponding author. E-mail: gotoxzh@163.com; jxnclcw@163.com

Received March 24, 2019; revised April 30, 2019; published online May 28, 2019.

Project supported by the Graduate Innovation Foundation of Jiangxi Province (No. YC2015-B023) and the Science and Technology Research Project of Jiangxi Provincial Education Department (No. GJJ170170).

江西省省研究生创新基金(No. YC2015-B023)和江西省教育厅科技攻关(No. GJJ170170)资助项目.
} 


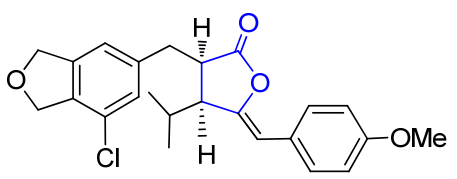

Cyanobacterin

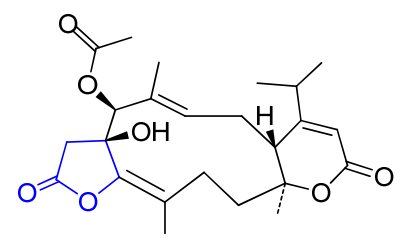

Atronone F<smiles>COC(NC(=O)C(=O)c1ccccc1)C1CC(c2ccccc2)C(=O)O1</smiles>

Growth regulating reagent<smiles>O=C1CCC(=C[C@H](O)[C@H](O)c2ccccc2)O1</smiles>

Goniobutenolides

Figure 1 Some examples of $\gamma$-alkylidene lactones frameworks

Previous work

(a) Conjugate addition of metalated teminal alkynes with Meldrum's acid derived acceptors
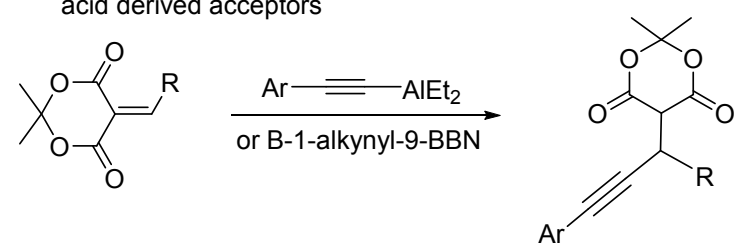

(b) Conjugate addition of in situ generated Cu-alkynylides with Meldrum's acid derived acceptors
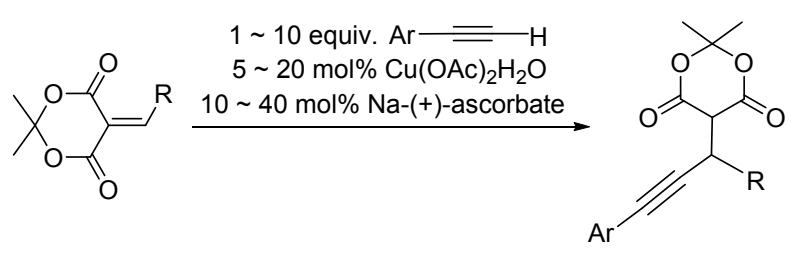

(c) Zinc-mediated conjugate addition of Alkynes to Meldrum's acid derived acceptors
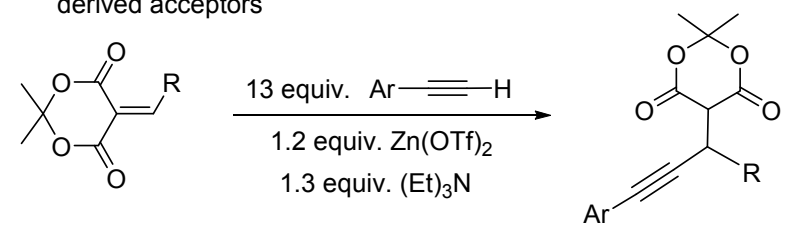

(d) Present work: three-component reaction
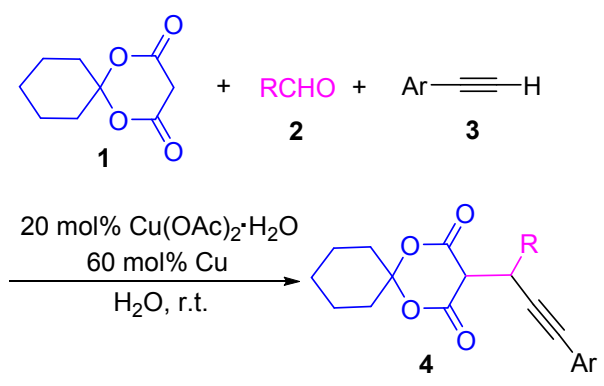

Scheme 1 Comparison between metalated teminal alkynes and in situ generated metal alkynlides

drum's acids (Scheme 1b). The method was optimal only for the addition of phenylacetylenes to $\gamma$-branched alkylidene acceptors. Fillion et al. ${ }^{[14]}$ developed a Rh-catalyzed addition to benzylidene acceptors and the scope was limited to trimethylsilylacetylene as the nucleophile. Walk$\mathrm{er}^{[15]}$ and Johnson et al. ${ }^{[16]}$ carried out the conjugate alkynylation of 5-benzylidene Meldrum's acid derivatives with alkynyl Grignard regents. Despite their many advantages, these processes have several unsatisfactories, such as harsh reaction conditions, low yields and the con- ditions of rigorous exclusion of oxygen and moisture. Carreira et $a{ }^{\left[{ }^{[17]}\right.}$ reported the diastereoselective alkynylation of chiral oxazepanedione acceptors with $\mathrm{Zn}(\mathrm{OTf})_{2}$ and amine base (Scheme 1c). The receptor substituents were also only limited to alkyl group. Hence, the development of some simple, wide substrates and efficient procedures is highly desirable.

Multicomponent reactions with the properties of easy operation, high efficiency, high selectivity and atomic economy are considered to be the closest to the ideal synthesis process and have been widely used in the fields of the total synthesis of natural products, heterocyclic compounds and combinatorial chemistry. ${ }^{[18]}$ Design of novel MCRS has been becoming a challenging objective. Our group has successfully prepared many heterocyclic compounds containing indole, spiro or dispiro structure by Yonemitsu $^{[19]}$ and Biginelli-like reactions. ${ }^{[20]}$ In continuation of our efforts toward the development of novel heterocyclic compounds, ${ }^{[21]}$ herein we would explore the synthesis of 5-(1-phenyl-3-phenylprop-2-ynyl)-2,2-pentylidene-1,3-dioxane-4,6-dionederivatives by a three-component reaction of aldehydes with 2,2-pentylidene-1,3-dioxane-4,6-dione and arylacetylene in the presence of $\mathrm{Cu}(\mathrm{OAc})_{2} / \mathrm{Cu}$ (Scheme 1d).

\section{Results and discussion}

To date, in a series of reported literatures, it was only found that $\beta$-alkynyl Meldrum's acids were prepared from in situ generated $\mathrm{Cu}$-catalyzed conjugate addition reaction of aromatic alkynes to Meldrum's acid derived accetors. In this paper, we document the example for the synthesis of 5-(1-phenyl-3-phenylprop-2-ynyl)-2,2-pentylidene-1,3-dioxane-4,6-dione derivatives by a three-component reaction. For optimization of conditions, 2,2-pentylidene-1,3-dioxane-4,6-dione (1), benzaldehyde (2a), and phenylacetylene (3a) were chosen as a model reaction (Eq. 1). The results are summarized in Table 1. In our initial screening experiments, the effects of the copper salts were examined. Different copper salts including $\mathrm{CuSO}_{4} \cdot 5 \mathrm{H}_{2} \mathrm{O}, \mathrm{CuCl}_{2} \bullet$ $2 \mathrm{H}_{2} \mathrm{O}, \mathrm{Cu}\left(\mathrm{NH}_{3}\right)_{4} \mathrm{SO}_{4}, \mathrm{Cu}_{2}\left(\mathrm{CO}_{3}\right)(\mathrm{OH})_{2}, \mathrm{Cu}(\mathrm{acac})_{2}, \mathrm{CuI}$, in situ generated $\mathrm{CuOAc}$ and $\mathrm{Cu}(\mathrm{OAc})_{2} \bullet \mathrm{H}_{2} \mathrm{O}$ were used (Table 1 , Entries $1 \sim 8) . \mathrm{Cu}(\mathrm{OAc})_{2} \cdot \mathrm{H}_{2} \mathrm{O}$ was found to be the most effective catalyst, leading to the desired product in $83 \%$ yield. In a blank experiment, the reaction did not occur in the absence of copper catalyst (Table 1, Entry 9). The influences of various reductants such as sodium ascorbate, $\mathrm{NH}_{2} \mathrm{OH} \cdot \mathrm{HCl}, \mathrm{Na}_{2} \mathrm{SO}_{3}$ and $\mathrm{Cu}$ on the model rea- 
ction were investigated. It was found that $\mathrm{Cu}$ was the best choice (Table 1, Entries 10 12). The effect of reaction time on the reaction was also examined and it was found that $10 \mathrm{~h}$ gave the best result (Table 1, Entries 13, 14). Therefore the optimized conditions are the use of a combination of $20 \mathrm{~mol} \% \mathrm{Cu}(\mathrm{OAc})_{2} \bullet \mathrm{H}_{2} \mathrm{O}$ as the catalyst and 60 $\mathrm{mol} \% \mathrm{Cu}$ as the reductant at room temperature for $10 \mathrm{~h}$.

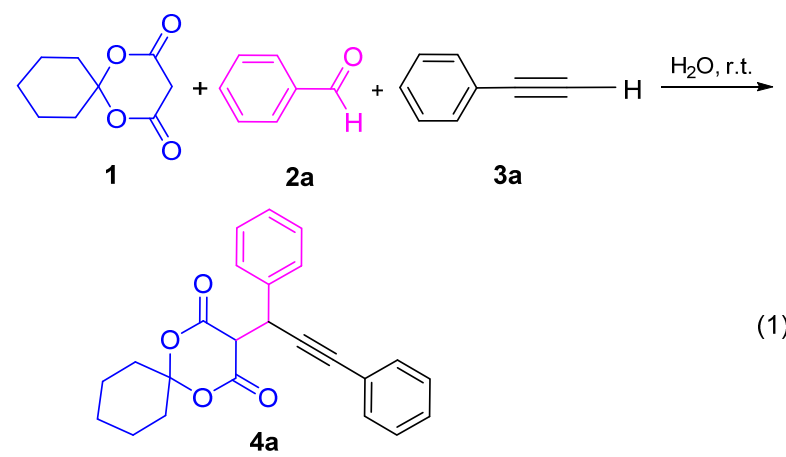

Our attention was then turned to the possibility of recycling excessive copper powder. After completion of the reaction, the reaction mixture was filtered. The residue treated with $\mathrm{CH}_{2} \mathrm{Cl}_{2}$ and washed by water, was recovered and then subjected to optimized conditions for the model reaction. The recycled copper powder as a reductant still showed a high activity and gave the corresponding product in high yield (Table 1, Entry 15).

Table 1 Optimization of the reaction conditions for the synthesis of 5-(1-phenyl-3-phenylprop-2-ynyl)-2,2-pentylidene-1,3-dioxane-4,6-dione (4a) ${ }^{a}$

\begin{tabular}{|c|c|c|c|c|}
\hline Entry & Copper salt & Reductant & Time/h & Yield $^{b} / \%$ \\
\hline 1 & $\mathrm{CuSO}_{4} \cdot 5 \mathrm{H}_{2} \mathrm{O}$ & $\mathrm{Cu}$ & 24 & 51 \\
\hline 2 & $\mathrm{CuCl}_{2} \cdot 2 \mathrm{H}_{2} \mathrm{O}$ & $\mathrm{Cu}$ & 24 & 39 \\
\hline 3 & $\mathrm{Cu}_{2}\left(\mathrm{CO}_{3}\right)(\mathrm{OH})_{2}$ & $\mathrm{Cu}$ & 24 & 12 \\
\hline 4 & $\mathrm{Cu}(\mathrm{acac})_{2}$ & $\mathrm{Cu}$ & 24 & 44 \\
\hline 5 & $\mathrm{Cu}\left(\mathrm{NH}_{3}\right)_{4} \mathrm{SO}_{4}$ & $\mathrm{Cu}$ & 24 & 31 \\
\hline $6^{c}$ & $\mathrm{CuI}$ & - & 20 & 0 \\
\hline $7^{d}$ & $\mathrm{CuOAc}$ & - & 10 & 56 \\
\hline 8 & $\mathrm{Cu}(\mathrm{OAc})_{2} \cdot \mathrm{H}_{2} \mathrm{O}$ & $\mathrm{Cu}$ & 10 & 83 \\
\hline $9^{c}$ & & $\mathrm{Cu}$ & 24 & 0 \\
\hline 10 & $\mathrm{Cu}(\mathrm{OAc})_{2} \cdot \mathrm{H}_{2} \mathrm{O}$ & Sodium ascorbate & 6 & 75 \\
\hline $11^{c}$ & $\mathrm{Cu}(\mathrm{OAc})_{2} \cdot \mathrm{H}_{2} \mathrm{O}$ & $\mathrm{NH}_{2} \mathrm{OH} \cdot \mathrm{HCl}$ & 6 & 0 \\
\hline 12 & $\mathrm{Cu}(\mathrm{OAc})_{2} \cdot \mathrm{H}_{2} \mathrm{O}$ & $\mathrm{Na}_{2} \mathrm{SO}_{3}$ & 6 & 53 \\
\hline 13 & $\mathrm{Cu}(\mathrm{OAc})_{2} \cdot \mathrm{H}_{2} \mathrm{O}$ & $\mathrm{Cu}$ & 8 & 71 \\
\hline 14 & $\mathrm{Cu}(\mathrm{OAc})_{2} \cdot \mathrm{H}_{2} \mathrm{O}$ & $\mathrm{Cu}$ & 12 & 83 \\
\hline $15^{e}$ & $\mathrm{Cu}(\mathrm{OAc})_{2} \cdot \mathrm{H}_{2} \mathrm{O}$ & $\mathrm{Cu}$ (recycled) & 10 & 76 \\
\hline
\end{tabular}

${ }^{a}$ Reaction conditions: reactions were conducted using $20 \mathrm{~mol} \% \mathrm{Cu}(\mathrm{II})$ salt, 1.5 equiv. of $\mathrm{PhC}=\mathrm{CH}$, and $60 \mathrm{~mol} \%$ reductant in $4 \mathrm{~mL}$ of $\mathrm{H}_{2} \mathrm{O}$ at room temperature; ${ }^{b}$ Isolated yield based on $\mathbf{2 a} ;{ }^{c}$ The products were 5,5 -(phenylmethylene)bis(2,2-pentylidene-1,3-dioxane-4,6-dione); ${ }^{d}$ the reaction conditions: CuOAc was synthesized from a solution of $20 \mathrm{~mol} \% \mathrm{Cu}(\mathrm{II})$ salt and $60 \mathrm{~mol} \%$ reductant in $4 \mathrm{~mL}$ of $\mathrm{H}_{2} \mathrm{O}$, subsequently 1.5 equiv. of $\mathrm{PhC}=\mathrm{CH}, 2.0$ equiv. of benzaldehyde and $1 \mathrm{mmol}$ of 2,2-pentylidene-1,3-dioxane-4,6-dione were added; ${ }^{e}$ the yield for the fifth time.

Under the optimized reaction conditions, 2,2-pentylidene-1,3-dioxane-4,6-dione and phenylacetylene were ini- tially used as substrates for exploring the scope of aldehyde substrates. Reactions of aliphatic aldehydes (acetaldehyde, $n$-butyl aldehyde) and benzaldehyde provided good yields between $60 \% \sim 83 \%$ (Table 2, Entries 1, 9, 10). And then, the substituent effects of aromatic aldehydes were tested to investigate the generality of the reaction. The results exhibited various para-substituted benzaldehydes with electron withdrawing groups $\left(\mathrm{F}, \mathrm{Cl}, \mathrm{NO}_{2}\right)$ and electron donating groups $\left(\mathrm{CH}_{3}, \mathrm{CH}_{3} \mathrm{O}\right)$ have given good to excellent yields (Table 2, Entries 2 8).

Table 2 Substrate scope of aldehydes and arylacetylene ${ }^{a}$

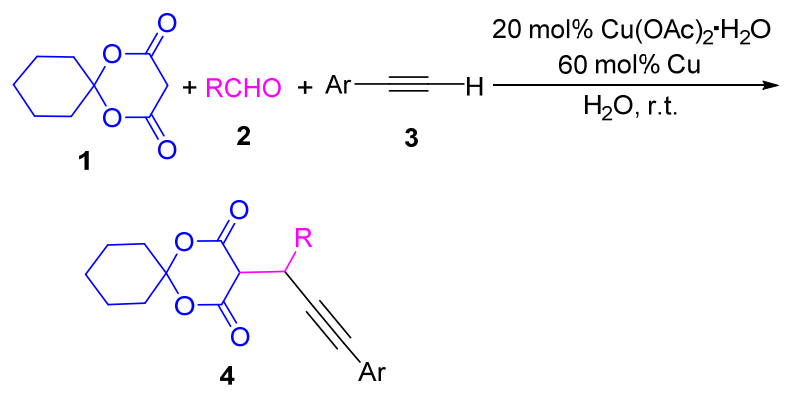

\begin{tabular}{cllccc}
\hline Entry & \multicolumn{1}{c}{$\mathrm{R}$} & \multicolumn{1}{c}{$\mathrm{Ar}$} & Time/h & Product & Yield $^{b} \%$ \\
\hline 1 & $\mathrm{C}_{6} \mathrm{H}_{5}$ & $\mathrm{C}_{6} \mathrm{H}_{5}$ & 10.0 & $\mathbf{4 a}$ & 83 \\
2 & $4-\mathrm{FC}_{6} \mathrm{H}_{4}$ & $\mathrm{C}_{6} \mathrm{H}_{5}$ & 8.0 & $\mathbf{4 b}$ & 69 \\
3 & $4-\mathrm{ClC}_{6} \mathrm{H}_{4}$ & $\mathrm{C}_{6} \mathrm{H}_{5}$ & 8.0 & $\mathbf{4 c}$ & 70 \\
4 & $4-\mathrm{CH}_{3} \mathrm{C}_{6} \mathrm{H}_{4}$ & $\mathrm{C}_{6} \mathrm{H}_{5}$ & 6.0 & $\mathbf{4 d}$ & 75 \\
5 & $4-\mathrm{NO}_{2} \mathrm{C}_{6} \mathrm{H}_{4}$ & $\mathrm{C}_{6} \mathrm{H}_{5}$ & 12.0 & $\mathbf{4 e}$ & 71 \\
6 & $4-\mathrm{CH}_{3} \mathrm{OC}_{6} \mathrm{H}_{4}$ & $\mathrm{C}_{6} \mathrm{H}_{5}$ & 8.0 & $\mathbf{4 f}$ & 66 \\
7 & $2-\mathrm{Furyl}$ & $\mathrm{C}_{6} \mathrm{H}_{5}$ & 6.0 & $\mathbf{4 g}$ & 85 \\
8 & $\mathrm{PhCH}=\mathrm{CH}_{9}$ & $\mathrm{C}_{6} \mathrm{H}_{5}$ & 12.0 & $\mathbf{4 h}$ & 88 \\
9 & $\mathrm{CH}_{3}$ & $\mathrm{C}_{6} \mathrm{H}_{5}$ & 20.0 & $\mathbf{4 i}$ & 60 \\
10 & $\mathrm{CH}_{3} \mathrm{CH}_{2} \mathrm{CH}_{2}$ & $\mathrm{C}_{6} \mathrm{H}_{5}$ & 20.0 & $\mathbf{4 j}$ & 63 \\
11 & $\mathrm{C}_{6} \mathrm{H}_{5}$ & $4-\mathrm{ClC}_{6} \mathrm{H}_{4}$ & 8.0 & $\mathbf{4 k}$ & 76 \\
12 & $\mathrm{CH}_{3} \mathrm{CH}_{2} \mathrm{CH}_{2}$ & $4-\mathrm{ClC}_{6} \mathrm{H}_{4}$ & 20.0 & $\mathbf{4 l}$ & 70 \\
\hline
\end{tabular}

${ }^{a}$ Reaction conditions: reactions were conducted using $20 \mathrm{~mol} \% \mathrm{Cu}(\mathrm{OAc})_{2}{ }^{\circ}$ $\mathrm{H}_{2} \mathrm{O}, 1.5$ equiv. of $\mathrm{ArC} \equiv \mathrm{CH}$, and $60 \mathrm{~mol} \% \mathrm{Cu}$ in $4 \mathrm{~mL}$ of $\mathrm{H}_{2} \mathrm{O}$ at room temperature; ${ }^{b}$ isolated yield.

In order to get further information on the intermediate form of the phenylethynyl-Cu(I) 6, after reduction of $\mathrm{Cu}(\mathrm{OAc})_{2} \cdot \mathrm{H}_{2} \mathrm{O}$ by $\mathrm{Cu}$ in water, phenylacetylene $(1.5$ equiv.) was added. The resulting mixture was stirred for 6 $\mathrm{h}$, then the mixture was extracted with $\mathrm{CH}_{2} \mathrm{Cl}_{2}$, dried over $\mathrm{Na}_{2} \mathrm{SO}_{4}$, concentrated under reduced pressure. The yellow residue obtained was washed by absolute alcohol and dried by vacuum. The yellow powder was subjected to infrared and mass spectroscopic analysis. In the high-resolution MALDI-TOF mass spectrum, the major peak corresponded to $(\mathrm{PhC} \equiv \mathrm{CCu}+\mathrm{Na})^{+} \mathrm{m} / \mathrm{z}$ 187.0. In infrared spectroscopy, the stretch frequencies of $\mathrm{C} \equiv \mathrm{C}$ bond decrease from 2120 $\mathrm{cm}^{-1}$ for phenylacetylene to $1929 \mathrm{~cm}^{-1}$ for the copper alkynylide. From the above results and literatures, ${ }^{[22,23]} \mathrm{a}$ reasonable mechanism for one-pot three-component synthesis of 5-(1-phenyl-3-phenylprop-2-ynyl)-2,2-pentylidene-1,3-dioxane-4,6-dione (4a) is depicted in Scheme 2. Firstly, the Knoevenagel reaction of benzaldehyde with 
2,2-pentylidene-1,3-dioxane- 4,6-dione would easily form the intermediate 5-phenyl-methylene-2,2-pentylidene-1,3dioxane-4,6-dione (5) in polar protic solvent. The terminal $\mathrm{C}-\mathrm{H}$ of phenylacetylene is activated by $\mathrm{Cu}(\mathrm{I})$ prepared from $\mathrm{Cu}(\mathrm{OAc})_{2} \cdot \mathrm{H}_{2} \mathrm{O}$ in the presence of $\mathrm{Cu}$, and then the phenylethynyl-Cu(I) 6 is formed. Subsequently, the product $\mathbf{4 a}$ is obtained by the conjugate addition reaction.
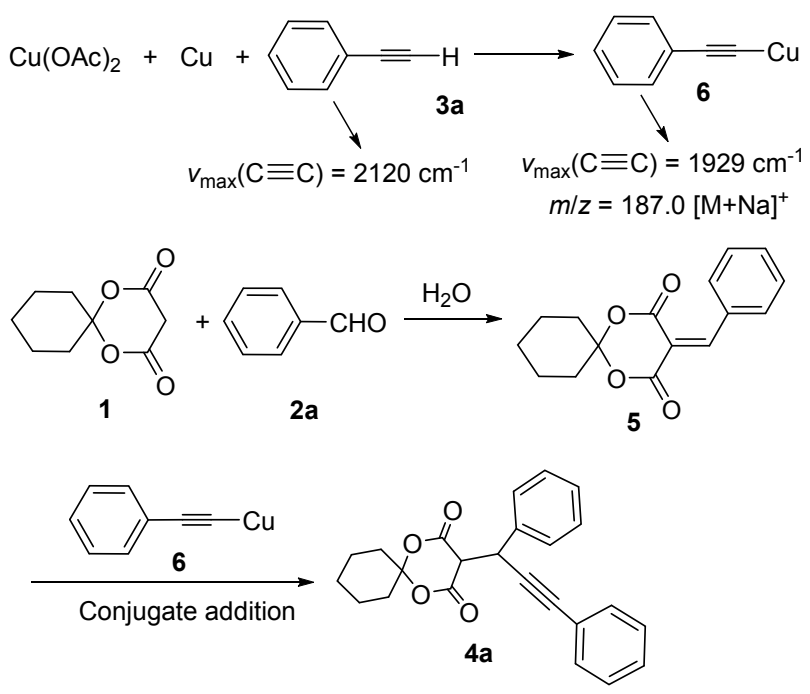

Scheme 2 Proposed mechanism for the imformation of $\mathbf{4 a}$

\section{Conclusions}

In summary, a combination of three-component synthesis of 5-(1-phenyl-3-phenylprop-2-ynyl)-2,2-pentylidene1,3-dioxane-4,6-dione derivtives catalyzed by $\mathrm{Cu}(\mathrm{OAc})_{2}{ }^{\bullet}$ $\mathrm{H}_{2} \mathrm{O}$ and $\mathrm{Cu}$ has been developed. The operation and workup procedures were very simple and nocolumn chrommatography purification was needed. This provides an effective method for the synthesis of $\beta$-alkynyl Meldrum's acid analogues.

\section{Experimental section}

\subsection{Chemical and instrument}

2,2-Pentylidene-1,3-dioxane-4,6-dione was prepared according to Ref. [24]. The other chemicals were purchased from Aladdin, Aldrich and Fluka Chemical Companies and without further purification. Melting points were measured on an XT-4 digital micro melting point apparatus and were uncorrected. ${ }^{1} \mathrm{H}$ NMR and ${ }^{13} \mathrm{C}$ NMR spectra were recorded on a Bruker AV-400 instrument with $\mathrm{CDCl}_{3}$ as solvent. The analytical MS of the compounds was performed on a Agilent LC-MS 6120.

4.2 General procedure of preparation of 5-(1-phenyl3-phenylprop-2-ynyl)-2,2-pentylidene-1,3-dioxane-4, 6-dione derivatives

To a $25 \mathrm{~mL}$ tube equiped with a stirring bar were added $\mathrm{H}_{2} \mathrm{O}(4.0 \mathrm{~mL}), \mathrm{Cu}(\mathrm{OAc})_{2} \cdot \mathrm{H}_{2} \mathrm{O}(0.2 \mathrm{mmol}, 20 \mathrm{~mol} \%)$, copper powder $(0.6 \mathrm{mmol}, 60 \mathrm{~mol} \%)$, arylacetylene $(3,1.5$ mmol), 2,2-pentylidene-1,3-dioxane-4,6-dione (1, $1 \mathrm{mmol})$ and aldehydes $(\mathbf{2} \mathbf{a} \sim \mathbf{2} \mathbf{j}, 1 \mathrm{mmol})$. The vessel was then sealed with a screw cap and the appropriate times were set at room temperature (Table 2). Upon completion of the reaction, the filtration residue was treated with $\mathrm{CH}_{2} \mathrm{Cl}_{2}$ and washed by water, and excessive copper was dried by under vacuum and then subjected to optimized conditions for the model reaction. The filtrate was treated with $\mathrm{CH}_{2} \mathrm{Cl}_{2}$ and saturated aqueous solution of $\mathrm{NH}_{4} \mathrm{Cl}$. The organic layer was separated and the water phase was extracted with $\mathrm{CH}_{2} \mathrm{Cl}_{2}(15 \mathrm{~mL} \times 2)$. The combined organic layers were dried over $\mathrm{Na}_{2} \mathrm{SO}_{4}$ and concentrated under reduced pressure. The residue was purified by recrystallization from absolute ethanol and drying to afford the desired compounds $\mathbf{4 a} \sim \mathbf{4 l}$.

\subsection{Spectral data of the products}

(1-Phenyl-3-phenylprop-2-ynyl)-2,2-pentylidene-1,3-dioxane-4,6-dione (4a): White solid, m.p. $126 \sim 128{ }^{\circ} \mathrm{C} .{ }^{1} \mathrm{H}$ NMR $\left(400 \mathrm{MHz}, \mathrm{CDCl}_{3}\right) \delta: 1.42 \sim 1.48(\mathrm{~m}, 2 \mathrm{H}), 1.64 \sim$ $1.71(\mathrm{~m}, 4 \mathrm{H}), 1.80(\mathrm{t}, J=5.6,6.4 \mathrm{~Hz}, 2 \mathrm{H}), 1.92(\mathrm{t}, J=5.6$, $4.9 \mathrm{~Hz}, 2 \mathrm{H}), 4.01(\mathrm{~d}, J=2.8 \mathrm{~Hz}, 1 \mathrm{H}), 5.15(\mathrm{~d}, J=2.8 \mathrm{~Hz}$, $1 \mathrm{H}), 7.27 \sim 7.38(\mathrm{~m}, 6 \mathrm{H}), 7.45 \sim 7.50(\mathrm{~m}, 2 \mathrm{H}), 7.62(\mathrm{~d}, J=$ $7.6 \mathrm{~Hz}, 2 \mathrm{H}) ;{ }^{13} \mathrm{C}$ NMR $\left(100 \mathrm{MHz}, \mathrm{CDCl}_{3}\right) \delta: 21.79,22.45$, $23.99,36.88,36.96,37.18,53.25,85.54,86.34,106.11$, $122.81,127.80,128.24,128.36,128.53,128.72,131.90$, 137.30, 163.22, 163.97; HRMS calcd for $\mathrm{C}_{24} \mathrm{H}_{22} \mathrm{NaO}_{4}$ $[\mathrm{M}+\mathrm{Na}]^{+}$397.1416, found 397.1422.

5-[1-(4-Flurophenyl)-3-phenylprop-2-ynyl]-2,2-pentylidene-1,3-dioxane-4,6-dione (4b): White solid, m.p. 119 $121{ }^{\circ} \mathrm{C} .{ }^{1} \mathrm{H}$ NMR $\left(400 \mathrm{MHz}, \mathrm{CDCl}_{3}\right) \delta: 1.44 \sim 1.49(\mathrm{~m}$, $2 \mathrm{H}), 1.65 \sim 1.73(\mathrm{~m}, 4 \mathrm{H}), 1.83(\mathrm{t}, J=5.6,6.4 \mathrm{~Hz}, 2 \mathrm{H}), 1.93$ $(\mathrm{t}, J=5.6,6.4 \mathrm{~Hz}, 2 \mathrm{H}), 3.99(\mathrm{~d}, J=2.8 \mathrm{~Hz}, 1 \mathrm{H}), 5.13(\mathrm{~d}$, $J=2.8 \mathrm{~Hz}, 1 \mathrm{H}), 7.01 \sim 7.07(\mathrm{~m}, 2 \mathrm{H}), 7.28 \sim 7.35(\mathrm{~m}, 3 \mathrm{H})$, $7.44 \sim 7.49(\mathrm{~m}, 2 \mathrm{H}), 7.57 \sim 7.62(\mathrm{~m}, 2 \mathrm{H}) ;{ }^{13} \mathrm{C}$ NMR $(100$ $\left.\mathrm{MHz}, \mathrm{CDCl}_{3}\right) \delta: 21.76,22.47,23.97,36.37,36.85,36.90$, $53.23,85.54,86.26,106.16,115.23,115.44,122.63$, $128.28,128.48,130.65(\mathrm{~d}, J=32.8 \mathrm{~Hz}), 131.87,132.90$ (d, $J=12.8 \mathrm{~Hz}), 161.10(\mathrm{~d}, J=246.5 \mathrm{~Hz}), 163.55,163.69$; HRMS calcd for $\mathrm{C}_{24} \mathrm{H}_{21} \mathrm{FNaO}_{4}[\mathrm{M}+\mathrm{Na}]^{+}$415.1322, found 415.1329.

5-[1-(4-Chlorophenyl)-3-phenyl-prop-2-ynyl]-2,2-pentylidene-1,3-dioxane-4,6-dione (4c): White solid, m.p. $115 \sim 117{ }^{\circ} \mathrm{C} .{ }^{1} \mathrm{H}$ NMR $\left(400 \mathrm{MHz}, \mathrm{CDCl}_{3}\right) \delta: 1.43 \sim 1.49$ $(\mathrm{m}, 2 \mathrm{H}), 1.64 \sim 1.73(\mathrm{~m}, 4 \mathrm{H}), 1.86(\mathrm{t}, J=5.6,6.4 \mathrm{~Hz}, 2 \mathrm{H})$, $1.93(\mathrm{t}, J=5.6,6.4 \mathrm{~Hz}, 2 \mathrm{H}), 3.99$ (d, $J=2.8 \mathrm{~Hz}, 1 \mathrm{H}), 5.12$ (d, $J=2.8 \mathrm{~Hz}, 1 \mathrm{H}), 7.28 \sim 7.33(\mathrm{~m}, 5 \mathrm{H}), 7.44 \sim 7.48(\mathrm{~m}$, 2H), $7.56(\mathrm{~d}, J=8.4 \mathrm{~Hz}, 2 \mathrm{H}) ;{ }^{13} \mathrm{C} \mathrm{NMR}\left(100 \mathrm{MHz}, \mathrm{CDCl}_{3}\right)$ $\delta: \quad 21.76,22.49,23.97,36.42,36.80,36.89,53.12,85.72$, $85.90,106.21,122.56,128.29,128.53,128.62,130.26$, $131.89,133.74,135.77,163.15,163.66$; HRMS calcd for $\mathrm{C}_{24} \mathrm{H}_{21} \mathrm{ClNaO}_{4}[\mathrm{M}+\mathrm{Na}]^{+}$431.1026, found 431.1020.

5-[1-(4-Methylphenyl)-3-phenyl-prop-2-ynyl]-2,2-pentylidene-1,3-dioxane-4,6-dione (4d): White solid, m.p. $115 \sim 117{ }^{\circ} \mathrm{C} .{ }^{1} \mathrm{H}$ NMR $\left(400 \mathrm{MHz}, \mathrm{CDCl}_{3}\right) \delta: 1.42 \sim 1.48$ $(\mathrm{m}, 2 \mathrm{H}), 1.64 \sim 1.71(\mathrm{~m}, 4 \mathrm{H}), 1.80(\mathrm{t}, J=5.6,6.4 \mathrm{~Hz}, 2 \mathrm{H})$, $1.91(\mathrm{t}, J=5.6,6.4 \mathrm{~Hz}, 2 \mathrm{H}), 2.34(\mathrm{~s}, 3 \mathrm{H}), 3.99(\mathrm{~d}, J=2.8$ $\mathrm{Hz}, 1 \mathrm{H}), 5.12$ (d, $J=2.8 \mathrm{~Hz}, 1 \mathrm{H}), 7.16(\mathrm{~d}, J=7.6 \mathrm{~Hz}, 2 \mathrm{H})$, $7.28 \sim 7.32(\mathrm{~m}, 3 \mathrm{H}), 7.44 \sim 7.50(\mathrm{~m}, 4 \mathrm{H}) ;{ }^{13} \mathrm{C}$ NMR $(100$ 
$\left.\mathrm{MHz}, \mathrm{CDCl}_{3}\right) \delta: 21.09,21.79,22.45,24.00,36.88,36.95$, $53.32,85.34,85.58,106.05,122.89,128.22,128.30$, $128.59,129.21,131.89,134.28,137.55,163.29,164.01$; HRMS calcd for $\mathrm{C}_{25} \mathrm{H}_{24} \mathrm{NaO}_{4}[\mathrm{M}+\mathrm{Na}]^{+} 411.1572$, found 411.1580 .

5-[1-(4-Nitrophenyl)-3-phenyl-prop-2-ynyl]-2,2-pentylidene-1,3-dioxane-4,6-dione (4e): Light yellow solid, m.p. $125 \sim 126{ }^{\circ} \mathrm{C} .{ }^{1} \mathrm{H}$ NMR $\left(400 \mathrm{MHz}, \mathrm{CDCl}_{3}\right) \delta: 1.46 \sim 1.51$ $(\mathrm{m}, 2 \mathrm{H}), 1.66 \sim 1.77(\mathrm{~m}, 4 \mathrm{H}), 1.91 \sim 1.98(\mathrm{~m}, 4 \mathrm{H}), 4.09(\mathrm{~d}$, $J=2.8 \mathrm{~Hz}, 1 \mathrm{H}), 5.24(\mathrm{~d}, J=2.8 \mathrm{~Hz}, 1 \mathrm{H}), 7.31 \sim 7.36(\mathrm{~m}$, $3 \mathrm{H}), 7.45 \sim 7.49(\mathrm{~m}, 2 \mathrm{H}), 7.83(\mathrm{~d}, J=8.8 \mathrm{~Hz}, 2 \mathrm{H}), 8.20(\mathrm{~d}, J$ $=8.8 \mathrm{~Hz}, 2 \mathrm{H}) ;{ }^{13} \mathrm{C} \mathrm{NMR}\left(100 \mathrm{MHz}, \mathrm{CDCl}_{3}\right) \delta: 21.74$, $22.53,23.95,36.54,36.69,36.84,52.94,84.73,86.50$, $106.49,122.14,123.59,128.38,128.82,129.91,131.92$, 132.13, 144.65, 147.47, 162.87, 163.42; HRMS calcd for $\mathrm{C}_{24} \mathrm{H}_{21} \mathrm{NNaO}_{6}[\mathrm{M}+\mathrm{Na}]^{+}$442.1267, found 442.1259.

5-[1-(4-Methoxylphenyl)-3-phenyl-prop-2-ynyl]-2,2-pentylidene-1,3-dioxane-4,6-dione (4f): Light yellow solid, m.p. 90 92 ${ }^{\circ} \mathrm{C} .{ }^{1} \mathrm{H}$ NMR $\left(400 \mathrm{MHz}, \mathrm{CDCl}_{3}\right) \delta: 1.42 \sim$ $1.48(\mathrm{~m}, 2 \mathrm{H}), 1.63 \sim 1.70(\mathrm{~m}, 4 \mathrm{H}), 1.79(\mathrm{t}, J=5.6,6.4 \mathrm{~Hz}$, $2 \mathrm{H}), 1.91(\mathrm{t}, J=5.6,6.4 \mathrm{~Hz}, 2 \mathrm{H}), 3.79(\mathrm{~s}, 3 \mathrm{H}), 3.98(\mathrm{~d}, J=$ $2.8 \mathrm{~Hz}, 1 \mathrm{H}), 5.10(\mathrm{~d}, J=2.8 \mathrm{~Hz}, 1 \mathrm{H}), 6.86 \sim 6.90(\mathrm{~m}, 2 \mathrm{H})$, $7.28 \sim 7.32(\mathrm{~m}, 3 \mathrm{H}), 7.44 \sim 7.49(\mathrm{~m}, 2 \mathrm{H}), 7.51 \sim 7.55(\mathrm{~m}$, $2 \mathrm{H}) ;{ }^{13} \mathrm{C} \mathrm{NMR}\left(100 \mathrm{MHz}, \mathrm{CDCl}_{3}\right) \delta: 21.79,22.45,23.99$, $36.54,36.89,36.93,53.33,55.30,85.19,86.86,106.05$, $113.86,122.89$, 128.24, 128.31, 129.20, 129.98, 131.87, $159.19,163.46,163.91$; HRMS calcd for $\mathrm{C}_{25} \mathrm{H}_{24} \mathrm{NaO}_{5}$ $[\mathrm{M}+\mathrm{Na}]^{+}$427.1521, found 427.1528.

5-(1-Furan-2-yl-3-phenyl-prop-2-ynyl)-2,2-pentylidene1,3-dioxane-4,6-dione (4g): Off-white solid, m.p. 128 $130{ }^{\circ} \mathrm{C} .{ }^{1} \mathrm{H}$ NMR $\left(400 \mathrm{MHz}, \mathrm{CDCl}_{3}\right) \delta: 1.47 \sim 1.52(\mathrm{~m}$, $2 \mathrm{H}), 1.68 \sim 1.79(\mathrm{~m}, 4 \mathrm{H}), 1.95 \sim 2.01(\mathrm{~m}, 4 \mathrm{H}), 4.21(\mathrm{~d}, J=$ $2.8 \mathrm{~Hz}, 1 \mathrm{H}), 5.15(\mathrm{~d}, J=2.8 \mathrm{~Hz}, 1 \mathrm{H}), 6.38(\mathrm{dd}, J=2.0,3.2$ $\mathrm{Hz}, 1 \mathrm{H}), 6.52(\mathrm{~d}, J=3.2 \mathrm{~Hz}, 1 \mathrm{H}), 7.27 \sim 7.34(\mathrm{~m}, 4 \mathrm{H})$, $7.43 \sim 7.47(\mathrm{~m}, 2 \mathrm{H}) ;{ }^{13} \mathrm{C} \mathrm{NMR}\left(100 \mathrm{MHz}, \mathrm{CDCl}_{3}\right) \delta$ : $21.78,22.54,24.03,31.52,36.72,36.86,49.96,83.91$, $84.43,106.13,108.47,110.89,122.43,128.23,128.55$, $131.99,141.90,150.19,162.65,163.58$; HRMS calcd for $\mathrm{C}_{22} \mathrm{H}_{20} \mathrm{NaO}_{5}[\mathrm{M}+\mathrm{Na}]^{+}$387.1208, found 387.1214.

5-(3-Phenyl-1-phenylethynyl-allyl)-2,2-pentylidene-1,3dioxane-4,6-dione (4h) Light yellow solid, m.p. 158 $160{ }^{\circ} \mathrm{C} .{ }^{1} \mathrm{H}$ NMR $\left(400 \mathrm{MHz}, \mathrm{CDCl}_{3}\right) \delta: 1.46 \sim 1.55(\mathrm{~m}$, $2 \mathrm{H}), 1.67 \sim 1.78(\mathrm{~m}, 4 \mathrm{H}), 1.96 \sim 2.00(\mathrm{~m}, 4 \mathrm{H}), 3.90(\mathrm{~d}, J=$ $2.8 \mathrm{~Hz}, 1 \mathrm{H}), 4.58 \sim 4.60(\mathrm{~m}, 1 \mathrm{H}), 6.52(\mathrm{dd}, J=8.0,16.0$ $\mathrm{Hz}, 1 \mathrm{H}), 6.82(\mathrm{~d}, J=16.0 \mathrm{~Hz}, 1 \mathrm{H}), 7.23 \sim 7.33(\mathrm{~m}, 6 \mathrm{H})$, $7.41 \sim 7.46(\mathrm{~m}, 4 \mathrm{H}) ;{ }^{13} \mathrm{C}$ NMR $\left(100 \mathrm{MHz}, \mathrm{CDCl}_{3}\right) \delta$ : $21.77,22.57,24.04,34.85,36.55,36.93,52.00,84.56$, $86.31,106.20,122.81,125.22,126.73,127.96,128.20$, $128.30,128.56,131.89,133.89,136.33,163.32,163.45$; HRMS calcd for $\mathrm{C}_{26} \mathrm{H}_{24} \mathrm{NaO}_{4}[\mathrm{M}+\mathrm{Na}]^{+} 423.1572$, found 423.1583.

5-(1-Methyl-3-phenyl-prop-2-ynyl)-2,2-pentylidene-1,3dioxane-4,6-dione (4i): White solid, m.p. $141 \sim 143{ }^{\circ} \mathrm{C} .{ }^{1} \mathrm{H}$ NMR $\left(400 \mathrm{MHz}, \mathrm{CDCl}_{3}\right) \delta: 1.47 \sim 1.53(\mathrm{~m}, 2 \mathrm{H}), 1.56(\mathrm{~d}$, $J=7.2 \mathrm{~Hz}, 3 \mathrm{H}), 1.68 \sim 1.79(\mathrm{~m}, 4 \mathrm{H}), 1.95 \sim 2.02(\mathrm{~m}, 4 \mathrm{H})$, $3.69(\mathrm{~d}, J=3.2 \mathrm{~Hz}, 1 \mathrm{H}), 3.83$ (ddd, $J=2.8,7.2,10.0 \mathrm{~Hz}$, $1 \mathrm{H}), 7.24 \sim 7.29(\mathrm{~m}, 3 \mathrm{H}), 7.37 \sim 7.41(\mathrm{~m}, 2 \mathrm{H}) ;{ }^{13} \mathrm{C} \mathrm{NMR}$ $\left(100 \mathrm{MHz}, \mathrm{CDCl}_{3}\right) \delta: 18.55,21.80,26.54,24.05,26.50$, $36.58,37.06,51.26,82.59$, 89.20, 106.01, 123.00, 128.08, 128.16, 131.79, 163.73, 163.97; HRMS calcd for $\mathrm{C}_{19} \mathrm{H}_{20} \mathrm{NaO}_{4}[\mathrm{M}+\mathrm{Na}]^{+}$335.1259, found 335.1268.

5-(1-Propyl-3-phenyl-prop-2-ynyl)-2,2-pentylidene-1,3dioxane-4,6-dione (4j): White solid, m.p.125 126 ${ }^{\circ} \mathrm{C} .{ }^{1} \mathrm{H}$ NMR (400 MHz, $\left.\mathrm{CDCl}_{3}\right) \delta: 0.99(\mathrm{t}, J=7.2 \mathrm{~Hz}, 3 \mathrm{H})$, $1.46 \sim 1.52(\mathrm{~m}, 3 \mathrm{H}), 1.60 \sim 1.78(\mathrm{~m}, 6 \mathrm{H}), 1.96 \sim 2.01(\mathrm{~m}$, $4 \mathrm{H}), 2.10 \sim 2.22(\mathrm{~m}, 1 \mathrm{H}), 3.62 \sim 3.75(\mathrm{~m}, 2 \mathrm{H}), 7.23 \sim 7.28$ $(\mathrm{m}, 3 \mathrm{H}), 7.36 \sim 7.42(\mathrm{~m}, 2 \mathrm{H}) ;{ }^{13} \mathrm{C} \mathrm{NMR}(100 \mathrm{MHz}$, $\left.\mathrm{CDCl}_{3}\right) \delta: 13.60,21.22,21.81,22.50,24.01,32.16,34.81$, $36.74,37.10,50.39,83.62,88.14,106.02,123.04,128.05$, $128.14,131.80,163.81,164.54$; HRMS calcd for $\mathrm{C}_{21} \mathrm{H}_{24} \mathrm{NaO}_{4}[\mathrm{M}+\mathrm{Na}]^{+}$363.1572, found 363.1556.

5-[1-Phenyl-3-(4-Chlorophenyl)-prop-2-ynyl)-2,2-pentylidene-1,3-dioxane-4,6-dione (4k): Light yellow solid, m.p.129 $130{ }^{\circ} \mathrm{C} .{ }^{1} \mathrm{H}$ NMR $\left(400 \mathrm{MHz}, \mathrm{CDCl}_{3}\right) \delta: 1.42 \sim$ $1.48(\mathrm{~m}, 2 \mathrm{H}), 1.64 \sim 1.71(\mathrm{~m}, 4 \mathrm{H}), 1.78(\mathrm{t}, J=5.6,6.4 \mathrm{~Hz}$, $2 \mathrm{H}), 1.92(\mathrm{t}, J=5.6,4.9 \mathrm{~Hz}, 2 \mathrm{H}), 4.02(\mathrm{~d}, J=2.8 \mathrm{~Hz}, 1 \mathrm{H})$, $5.13(\mathrm{~d}, J=2.8 \mathrm{~Hz}, 1 \mathrm{H}), 7.27 \sim 7.41(\mathrm{~m}, 7 \mathrm{H}), 7.60(\mathrm{~d}, J=$ $7.2 \mathrm{~Hz}, 2 \mathrm{H}) ;{ }^{13} \mathrm{C}$ NMR (100 MHz, $\left.\mathrm{CDCl}_{3}\right) \delta: 21.78,22.47$, 23.97, 36.76, 36.89, 53.14, 53.19, 84.29, 84.72, 106.19, $121.28,127.92,128.60,128.73,133.15,134.41,137.03$, 163.142, 163.88; HRMS calcd for $\mathrm{C}_{24} \mathrm{H}_{21} \mathrm{ClNaO}_{4}[\mathrm{M}+$ $\mathrm{Na}]^{+}$431.1026, found $m / z 431.1033$.

5-[1-Propyl-3-(4-Chlorophenyl)-prop-2-ynyl]-2,2-pentylidene-1,3-dioxane-4,6-dione (4I): White solid, m.p. $127 \sim 128{ }^{\circ} \mathrm{C} .{ }^{1} \mathrm{H}$ NMR $\left(400 \mathrm{MHz}, \mathrm{CDCl}_{3}\right) \delta: 0.99(\mathrm{t}, J=$ $7.2 \mathrm{~Hz}, 3 \mathrm{H}), 1.42 \sim 1.51(\mathrm{~m}, 3 \mathrm{H}), 1.58 \sim 1.78(\mathrm{~m}, 6 \mathrm{H})$, $1.97 \sim 1.99(\mathrm{~m}, 4 \mathrm{H}), 2.08 \sim 2.19(\mathrm{~m}, 1 \mathrm{H}), 3.64 \sim 3.70(\mathrm{~m}$, $2 \mathrm{H}), 7.24(\mathrm{~d}, J=8.4 \mathrm{~Hz}, 2 \mathrm{H}), 7.31(\mathrm{~d}, J=8.4 \mathrm{~Hz}, 2 \mathrm{H}) ;{ }^{13} \mathrm{C}$ NMR $\left(100 \mathrm{MHz}, \mathrm{CDCl}_{3}\right) \delta: 13.63,21.25,21.79,22.53$, $24.03,31.92,34.60,36.60,36.99,50.34,82.41,89.24$, $106.07,121.53,128.49,133.05,134.05,163.72,164.40$; HRMS calcd for $\mathrm{C}_{21} \mathrm{H}_{23} \mathrm{ClNaO}_{4}[\mathrm{M}+\mathrm{Na}]^{+}$397.1183, found 397.1175 .

Supporting Information NMR spectra of $\mathbf{4 a} \sim \mathbf{4 l}$ is available free of charge via the Internet at http://siocjournal.cn/.

\section{References}

[1] (a) Bharate, S. B.; Nemmani, K. V. S.; Vishwakarma, R. A. Expert Opin. Ther. Pat. 2009, 19, 237.

(b) Nahm, S.; Weinreb, S. M. Tetrahedron Lett. 1981, 22, 3815.

[2] Knopfel, T. F.; Carreira, E. M. J. Am. Chem. Soc. 2003, 125, 6054.

[3] (a) Liu, S.; Fillion, E. Org. Lett. 2014, 16, 5748.

(b) Jia, W.; Li, S.; Yu, M.; Chen, M.; Jiao, N. Tetrahedron Lett. 2009, 50, 5406.

(c) Li, S.; Jia, W.; Jiao, N. Adv. Synth. Catal. 2009, 351, 569.

[4] Lee, E. J.; Gleason, F. K. Plant Sci. 1994, 103, 155.

[5] Edwards, K. R. PCT Int. Appl. 1982, 19.

[6] Huneck, S.; Schreiber, K. Phytochemistry 1972, 11, 2429.

[7] Fang, X.; Anderson, J. E.; Chang, C.; McLaughlin, J. L. Tetrahedron 1991, 47, 9751.

[8] (a) Puchot, C.; Samuel, O.; Dunach, E.; Zaho, S.; Agami, C.; Kagan, H. B. J. Am. Chem. Soc. 1986, 108, 2353.

(b) Sinclair, J. A.; Molander, G. A.; Brown, H. C. J. Am. Chem. Soc. 1977, 99, 954 . 
[9] (a) Schwartz, J.; Carr, D. B.; Hansen, R. T.; Dayrit, F. M. J. Org. Chem. 1980, 45, 3053.

(b) Bruhn, M.; Brown, C. H.; Collins, P. W.; Palmer, J. R.; Dajani, E. Z.; Pappo, R. Tetrahedron Lett. 1976, 17, 235.

[10] (a) Hooz, J.; Layton, R. B. J. Am. Chem. Soc. 1971, 93, 7320. (b) Schwartz, J.; Hansen, R. T.; Dayrit, F. M. J. Org. Chem. 1980, 45, 3053.

[11] (a) Kim, S.; Lee, J. M. Tetrahedron Lett. 1990, 31, 7627.

(b) Kim, S.; Park, J. H.; Jon, S. Y. Bull. Korean Chem. Soc. 1995, 16,783 .

[12] (a) Knopfel, T. F.; Zarotti, P.; Ichikawa, T.; Carreira, E. M. J. Am. Chem. Soc. 2005, 127, 9682.

(b) Fujimori, S.; Knopfel, T. F.; Zarotti, P.; Ichikawa, T.; Carreira, E. M.; Boyall, D. Bull. Chem. Soc. Jpn. 2007, 80, 1635.

(c) Fujimori, S.; Carreira, E. M. Angew. Chem., Int. Ed. 2007, 46, 4964.

(d) Zarotti, P.; Knopfel, T F.; Aschwanden, P.; Carreira, E. M. ACS Catal. 2012, 2, 1232.

[13] Mishra, S.; Liu, J.; Aponick, A. J. Am. Chem. Soc. 2017, 139: 3353.

[14] Fillion, E.; Zorzitto, A. K. J. Am. Chem. Soc. 2009, 131, 14608.

[15] Cui, S.; Walker, S. D.; Woo, J. C. S.; Borths, C. J.; Mukherjee, H.; Chen, M. J.; Faul, M. M. J. Am. Chem. Soc. 2010, 132, 436.

[16] Krabbe, S. W.; Do, D. T.; Johnson, J. S. Org. Lett. 2012, 14, 5932.

[17] Knopfel, T. F.; Boyall, D.; Carreira, E. M. Org. Lett. 2004, 6, 2281.

[18] (a) Xu, Z. H. Chin. J. Org. Chem. 2014, 34, 1687 (in Chinese). (许招会，有机化学, 2014, 34, 1687.)

(b) Yang, D. L.; Li, J. R.; Sun, K. N. Chin. J. Org. Chem. 2013, 33,
2341 (in Chinese).

(杨德利, 李加荣, 孙克宁, 路红燕, 刘明星, 史大昕, 有机化学, 2013, 33, 2341.)

(c) Francesco, E.; Salvatore, G.; Ornelio, R. Tetrahedron Lett. 2011, $59,568$.

[19] Lin, C. H.; Xu, Z. H.; Liao, W. L.; Qiu, Z. Y.; Xia, J. H. Chin. J. Org. Chem. 2015, 35, 212 (in Chinese)

(林春花, 许招会, 廖维林, 邱曾烨, 夏剑辉, 有机化学, 2015, 35,212 .)

[20] (a) Xu, Z. H.; Zhang, H. F.; Xiong, Y. K.; Liu, D. Y.; Huang, Q. S. Heterocycles 2016, 92, 2252.

(b) Xu, Z. H.; Zhang, H. F.; Lin, C. H.; Liu, D. Y. Heterocycles 2016, 92, 1031.

(c) Zhou, P.; Xu, Z. H. J. Jiangxi Normal Univ. (Nat. Sci.) 2019, 43, 167 (in Chinese).

(周鹏，许招会，江西师范大学学报(自然科学版), 2019, 43, 167.)

[21] Xu, Z. H.; Chen, F. B.; Li, Y. Y.; Huang, Q. S.; Liao, C. W. Chin. J. Org. Chem. 2018, 38, 3101 (in Chinese).

(许招会, 陈飞彪, 李瑜钰, 黄清水, 廖传文, 有机化学, 2018, 38, 3101.)

[22] Coates, G. E, Prakin, C. J. Inorg. Nucl. Chem. 1961, 22, 59.

[23] Manna, J.; John, K. D.; Hopkins, M. D. Adv. Organomet. Chem. 1995, 38, 79.

[24] Yan, N.; Xiong, B.; Liao, W. L.; Xu, Z. H. Chin. J. Org. Chem. 2010, 30, 1391 (in Chinese).

(严楠, 熊斌, 廖维林, 许招会, 有机化学, 2010, 30, 1391.)

(Li, L.; Fan, Y.) 